\title{
La apropiación de la educación virtual por parte de los colectivos sociales: las nuevas relaciones entre la tecnología, el conocimiento, y lo social
}

\author{
Bety Díaz Subieta ${ }^{1}$, Maynor Barrientos Amador $^{2}$ \\ ${ }^{1}$ Universidad Católica Luis Amigó Estatal - Colombia. Facultad de Psicología y Ciencias Sociales, Programa de Psicología. \\ Red Internacional de Investigadores en Educación a Distancia en Línea y Abierta (REDIC). Avenida Suba 128 A - 51. \\ Apartado postal. Bogotá 11. Bogotá, Colômbia. ${ }^{2}$ Universidad Estatal a Distancia de Costa Rica - UNED. Apartado postal San \\ José 474-2050. San José Costa Rica. \\ Autor para correspondência/Author for correspondence: luz.diazsu@amigo.edu.co / mbarrientos@uned.ac.cr
}

RESUMEN. Las nuevas "crisis educativas" no solo tienen como punto de partida los debates a lo interno de la filosofía y la psicología (conocimiento), la política, el poder y la pedagogía (aprendizaje), o a lo interno de las ciencias sociales y la tecnología (tecnología y sociedad), sino que surgen de los profundos cambios en las formas de interacción entre los seres humanos en determinados espacios sociales multifacéticos y complejos. Es cada vez más evidente que los retos educativos del futuro no son dictados por las instituciones educativas, sino por los colectivos sociales diversos y cómo ellos se apropian de sus propios conceptos, formas y objetivos educativos. Qué aprender, cómo hacerlo y para qué hacerlo son preguntas que surgen ahora de los grupos sociales en transformación y no desde los estados que los gobiernan. Así las cosas, es la educación virtual la modalidad educativa con mayores posibilidades de llegar a ser el modelo que mejor responde a las necesidades de dichos colectivos. Este trabajo pretende sugerir cuáles son los puntos de encuentro entre el modelo educativo virtual y la forma de interacción utilizada por los nuevos colectivos que motivará el panorama educativo del futuro.

Palabras clave: Congreso, Pedagogía, Colectivo Social, Educación Virtual. 


\title{
The appropriation of virtual education by social collectives: the new relations between technology, knowledge and social
}

\begin{abstract}
The so-called "educational crises" do not only surge from the internal debates in philosophy and psychology (knowledge), politics, power and pedagogy (learning), or inside the social sciences and the advance of technology (technology and society), but also these crises originate from the deep changes in the ways in which human beings interact within determined complex and multifaceted social spaces. It has become evident that future educational challenges are not defined by educational institutions, but by diverse social groups, and by how they dictate their own concepts, forms and educational objectives. What to learn, how to do it and why do it are all questions that find their answers within the social groups that have themselves been adapting, and not from the governmental institutions. Therefore, virtual education has big possibilities of becoming the modality that could best fit the needs of these groups. This work points to the intersection between virtual education and the way in which social groups interact and that will modify the educational scenes of the future.
\end{abstract}

Keywords: Conference, Pedagogy, Online Education, Social Collectives. 


\section{A apropriação da educação virtual por parte dos coletivos sociais: as novas relações entre a tecnologia, o conhecimento e o social}

RESUMO. As novas "crises educacionais" não têm apenas como ponto de partida os debates em filosofia e psicologia (conhecimento), política, poder e pedagogia (aprendizagem), ou dentro das ciências sociais e tecnológicas. (tecnologia e sociedade), mas surgem das profundas mudanças nas formas de interação entre os seres humanos em certos espaços sociais complexos e multifacetados. É cada vez mais evidente que os desafios educacionais do futuro não são ditados pelas instituições educacionais, mas por diversos grupos sociais e como eles se apropriam de seus próprios conceitos, formas e objetivos educacionais. O que aprender, como fazer e por que fazer são perguntas que surgem agora dos grupos sociais em transformação e não dos estados que as governam. Assim, a educação virtual é a modalidade educacional com maiores possibilidades de se tornar o modelo que melhor responde às necessidades desses grupos. Este artigo pretende sugerir quais são os pontos de encontro entre o modelo educacional virtual e a forma de interação utilizada pelos novos grupos que motivarão o panorama educacional do futuro.

Palavras-chave: Congresso, Pedagogia, Coletivo Social, Educação Virtual. 


\section{Introducción}

Tradicionalmente, los debates acerca de la naturaleza del conocimiento, o sobre las posibilidades reales de conocer, han surgido desde la filosofía, las ciencias cognitivas, la psicología, las neurociencias, en suma, desde el campo de experimentación científica y pensamiento académico. Desde la Filosofía actual, Azcárraga (2002), en relación con el problema del conocimiento en las sociedades contemporáneas, insiste en la relevancia de preguntas tales como: ¿cómo podemos conocer?, ¿cuál es la importancia del conocimiento?, ¿qué hace feliz al ser humano contemporáneo? entre otras.

Pero no solo la dimensión epistemológica se presenta en crisis. Igualmente, la dimensión ontológica del ser humano en relación con lo social, lo político y los valores se han visto interrogados por los acelerados cambios tecnológicos y científicos, cambios que, en buena medida, son vistos como fines en sí mismos, sin corresponder de la misma manera a dimensiones humanas tales como el pensamiento crítico, la ética, o el bienestar del ser humano, en general. En el cruce de ambas, sin duda, las ciencias educativas han sido directamente afectadas por dichas "crisis" debido al papel central que juegan éstas en la conformación de las subjetividades de hoy.
Precisamente es a partir del impacto que han tenido estos debates en la pedagogía, y cómo la pedagogía se ha adaptado a la presión del cambio tecnológico, que comenzamos a observar, desde la práctica de enseñar y aprender, la preocupación por el conocimiento, su generación y su aplicación, y un nuevo espacio de reflexión y práctica. Ese nuevo espacio se encuentra a lo interno de los nuevos colectivos sociales emergentes.

Es indudable la intrínseca conexión entre conocimiento, educación y el movimiento Ciencia, Tecnología y Sociedad (CTS) que surgió en Europa a partir de la década de los 30. En la actualidad dicho movimiento se caracteriza por el papel preponderante que han tomado las Tecnologías de la Información y la Comunicación (TIC) como mediadoras de las interacciones entre los seres humanos y los colectivos sociales, sobre todo en el campo educativo, la enseñanza, el aprendizaje y la gestión del conocimiento. Algo clave a rescatar de dicho movimiento es que su origen se dio a partir de profundas reflexiones éticas sobre el papel de la Ciencia, la tecnología y la innovación y si estas aportan reales y viables soluciones a las diferentes problemáticas humanas. Sin embargo, en la actualidad, no son los cambios tecnológicos y sus aplicaciones educativas los que marcan la 
forma en que los seres humanos deben aprender. Hoy, son las formas de relaciones sociales emergentes las que, empoderándose del cambio tecnológico, han introducido las innovaciones educativas. El qué aprender, cómo, en qué momento y con qué nuevos propósitos son ahora preguntas que surgen, no del currículo educativo, sino de las nuevas interacciones sociales.

Las sociedades del conocimiento, determinadas por el acceso a la información, el avance y el uso cotidiano de las tecnologías, son cada vez más colaborativas y su organización cada vez más basada en las dinámicas de interacción en red. Desde la educación, esta organización ha tomado la figura de comunidades de aprendizaje que, de acuerdo con Celaya (2008), se relacionan con la unión de diferentes personas en torno a temas específicos con claridades metodológicas y objetivos de aprendizaje bien enfocados. De este modo se da un paso de apropiación del conocimiento de unas cuantas manos a todas estas redes sociales de conocimiento, esto es, hacia toda una vinculación y activa participación social.

Por su parte, los colectivos sociales han dado un paso más allá y han entendido su papel como innovadores pedagógicos, llevando la aplicación de la tecnología social a espacios inexplorados. Por primera vez, los académicos de la tecnología y la educación han tomado modelos de interacción social para sus innovaciones educativas, ya no desde lo debates filosóficos de otrora.

Una paradoja es el hecho de que la generación de conocimiento sigue siendo muy dependiente de intereses políticos y económicos, y que es precisamente de estas esferas desde donde surgen las principales decisiones y acciones para el desarrollo de Ciencia y estrategias hacia la generación de conocimiento, incluyendo lo referente a las TIC. Sin embargo, paralelamente, a través de las redes sociales y otros fuertes movimientos, los diferentes colectivos se están movilizando en una generación de conocimiento social que pertenece más a la sociedad y menos a los intereses económicos y políticos, aunque todavía falta mucho en este camino, ya son claros algunos logros en este sentido. La participación social está cobrando cada vez mayor importancia en la generación de conocimiento con una mirada que cobra fuerza respecto al uso ético, tanto de las tecnologías, como de la información y el conocimiento mismo. Esto a su vez impacta, en un efecto bumerán, a las preguntas filosóficas establecidas anteriormente desde la epistemología y la ontología. 
La nueva crisis del conocimiento se está gestando, por consiguiente, no solo desde lo científico tecnológico, sino desde lo social, lo que plantea nuevas interrogantes sobre diferentes problemáticas humanas, y ello implica diversas y divergentes reflexiones del orden filosófico, antropológico, político y social que muy bien Fabronni y Pinto (2006) nos ponen de manifiesto, desde las tendencias que los acelerados y diversos cambios de los últimos años en la humanidad están creando.

Podemos dar cuenta del profundo cambio que ha tenido la humanidad en los últimos años debido a diferentes fenómenos como: la globalización, las TIC, y los profundos cambios geográficos, ambientales, demográficos y geopolíticos. Las culturas son transformadas y están permeadas casi en su totalidad por las diferentes tecnologías y la virtualidad, transformando de modo radical las formas de aprender, de hacer Ciencia, de pensar y de gestionar el conocimiento. El acceso a la información no solo es mucho más rápido sino más amplio, ya que se puede acceder a una cantidad cada vez mayor de información en tiempo real, y acceder a información que antes no estaba disponible con facilidad; ya no existen barreras de tiempo ni espacio; pero a la vez, el acceso ilimitado requiere de las personas un desarrollo mayor de habilidad de gestión de la información. El impacto cultural es contundente, una persona puede tener diferentes referentes con respecto a lo que quiere, a quién es, porqué comparte diversos aspectos de manera global.

Un punto clave para la generación de conocimiento hoy es tocado por un artículo de la Revista de Tecnología Social (2011), denominado ¿Qué es la tecnología social? el cual enfatiza la preponderancia actual que cobra la participación social en la adecuada apropiación del conocimiento y la tecnología incluyendo desde lo comunitario las acciones participativas concretas, compromiso social y posturas éticas de los colectivos frente a la generación y uso de dichos conocimientos y de la tecnología, la creación de políticas públicas jalonadas por los propios ciudadanos en los diferentes países, entre otras muchas estrategias. Esto supone, a su vez, un impacto en la forma y condiciones de los aprendizajes. Esta renovada generación y gestión del conocimiento surge del diálogo entre las disciplinas, experiencias particulares, la nueva información, la Ciencia, el avance tecnológico, la pedagogía, pero especialmente desde el poder de la organización social de base. 
En esa inversión de valores, la educación virtual se sitúa en un lugar privilegiado.

\section{La educación virtual y su papel en la gestión de conocimiento a partir de los colectivos sociales}

Existe una premisa que parece ser la que mejor describe nuestros tiempos: "A un ritmo vertiginoso todo ha cambiado y todo se reinventa" (González, 2014). Dados los cambios antes mencionados en los campos disciplinares relacionados con la naturaleza del conocimiento, nuevos procesos de subjetivación, cambios en las dinámicas sociales, económicas y culturales, la forma de comunicarnos, educarnos, relacionarnos y vivir, hoy es muy diferente a la de los siglos anteriores; evidentemente la educación está teniendo que hacer un importante y radical giro, tanto en sus paradigmas, teorías, así como en sus concepciones del estudiante, en sus metodologías y didácticas. A la educación se le imponen actualmente nuevas necesidades y cambios profundos que respondan a las múltiples crisis de la realidad, nuevas maneras de enseñar que guíen a quienes aprenden por diferentes y creativos caminos, el nuevo educando y los vertiginosos ritmos del contexto así lo exigen. Estén o no preparadas las instituciones de Educación Superior, la formación disruptiva empieza a abrirse camino cada vez con mayor fuerza, jalonada profundamente desde los colectivos sociales y sus necesidades, miradas y expectativas de futuro.

Sin lugar a dudas, la gran reforma ha surgido del interior de la educación a distancia, (hacia otros modelos educativos, incluyendo los más tradicionales) por ser esta modalidad educativa la de mayor convergencia tecnológica, y el escenario desde donde emerge la educación virtual. Como era de esperarse, estos cambios trajeron consigo importantes implicaciones pedagógicas y fuertes exigencias en la formación docente, especialmente para afrontar el reto de la virtualidad donde su rol, otrora transmisionista, ahora orientador, mediador, requiere competencias de alto nivel. En consecuencia, el salto a la virtualidad no puede asumirse como un experimento social e institucional, o una simple transformación de los diferentes modelos mentales que se requieren promover entre los estudiantes para lograr aprendizajes significativos empleando entornos virtuales. Esta innovación debe considerarse como un hecho de relevancia educativa, para que permanezca, haciendo que las modalidades e-Learning y $b$ Learning sean estrategias que contribuyan efectivamente al logro de la visión de las Universidades, pero más importante es que 
desde los objetivos de desarrollo sostenible de la agenda 20 - 30, aporten nuevos y efectivos espacios para el abordaje de la compleja realidad. "Esta profunda evolución ha sido debida, en gran medida, a la incorporación en la escuela de elementos que facilitan una nueva creación, interpretación y, sobre todo, un nuevo acceso a la información y a la generación de conocimientos. Este proceso de cambio viene marcado por la utilización de Tecnologías de la Información y Comunicación (TIC)”. (Burgos, 2011, p. 17).

De acuerdo con Duart y Sangrá (2000), la educación virtual es el espacio asincrónico en el que se produce, de forma real, el proceso de aprendizaje gracias a las tecnologías de la comunicación y la información. Se puede decir que el $e-$ learning es una modalidad formativa enmarcada dentro de la denominada educación a distancia y que se refiere, en definitiva, a un proceso de enseñanza y aprendizaje mediado por la tecnología que puede darse de forma sincrónica o asincrónica. Por otra parte, Collis, (2001) comprende por educación virtual, los procesos formativos que no son guiados directamente por la presencia de un profesor en el aula, pero que sí incluyen la planeación y orientación de docentes a través de un medio de comunicación tecnológico que permite el desarrollo de la interrelación docente-estudiante.

Lo verdaderamente relevante de la educación virtual y a distancia en nuestros tiempos es que se concibe como el desarrollo de procesos formativos a través de sistemas de comunicación impresos y electrónicos o personas que participan en un proceso de aprendizaje en lugares y horarios asincrónicos y diferentes a las aulas de clase y a espacios físicos, están dados desde un ambiente virtual de aprendizaje, es decir que tienen como base el aprendizaje colaborativo, en red y están atravesados indudablemente por los colectivos sociales; como lo indican Suárez y Jardei (2008): "El uso de las Tecnologías de la Información y la Comunicación en los ambientes de aprendizaje no solamente nos da la opción de referirnos a Ambientes Virtuales de Aprendizaje, sino que de acuerdo a Luhmann, y es evidente, nos genera una mayor complejidad en las comunicaciones que se dan en los diferentes actores, situación que debe ser abordada para evitar que sea un trauma el uso de éstos ambientes". (p.6).

El enfoque de educación virtual y a distancia, encaminado pertinentemente con las necesidades contextuales, pueden responder muy bien a los movimientos de los colectivos sociales, puesto que la 
inclusión de TIC en educación aporta facilidades de espacio, locación geográfica, tiempo, optimización de recursos tanto para estudiantes como docentes y actores de los sistemas formativos, permitiendo mayor cobertura, centrando la mirada en el autoaprendizaje y autorregulación del proceso por parte del estudiante $\mathrm{y}$ transformando claramente el rol del docente, ahora más de guía y orientador. (Silvio, 2000). Este es un punto relevante, puesto que de esta manera el conocimiento y el aprendizaje dejan de estar dictados única y exclusivamente desde la educación formal y dan paso a procesos mucho más sociales y en red, que tienen sus propias dinámicas de autorregulación y de co construcción.

De lo mencionado arriba, se concluye que, al superarse el tema de espacios físicos e infraestructuras, y las condiciones excluyentes para la formación de un alto número de personas, que por diferentes razones no pueden estudiar de manera presencial, se ganan espacios diferentes de conocimiento que facilitan el acceso de grandes colectivos a programas de formación que respondan a sus necesidades particulares, sean estas de tipo laboral, de movilización, sociales e incluso económicas. Becerra (2002) indica que, si una propiedad caracteriza de modo distintivo a la educación virtual respecto de los entornos presenciales, pero también respecto de las modalidades tradicionales de educación a distancia, es precisamente la posibilidad de interacción con autonomía del tiempo y de las distancias. Esta posibilidad es definida como asincrónica, ya que este elemento es parte fundamental del componente formativo virtual, permitiendo manejar lapsos de tiempo en la comunicación que van desde los modelos comunicativos en línea, en tiempo real hasta el logro de programaciones específicas de actividades de aprendizaje que permiten a muchas personas interactuar con docentes virtuales, especialistas formativos, o entre los mismos estudiantes de manera efectiva determinando lapsos de tiempo específicos entre los aportes y las retroalimentaciones, que se constituyen en verdaderas comunidades de aprendizaje.

En lo relacionado con las TIC como herramientas de comunicación hoy, Sánchez (2008) las define como plataformas de transmisión de información, que conectan comunidades y generan redes sociales. Esto hace que tanto la academia como el mundo empresarial las utilicen en sus operaciones diarias, como facilitadoras de conocimiento a través del desarrollo de nuevas metodologías de aprendizaje con presentaciones novedosas de los 
contenidos y actividades a desarrollar por parte de quien aprende.

\section{El rol del colectivo como agente transformador de la educación virtual}

Respecto a las nuevas formas de aprender y las interrelaciones de los estudiantes en el marco de la inclusión de TIC en la educación, en primera instancia es pertinente retomar el hecho de que el aprendizaje es un proceso mediado por la socialización, de acuerdo a Vigostky (1979), quien plantea el desarrollo cognitivo y el aprendizaje a partir del contacto con el mundo social para luego pasar a internalizarse. El estudiante aprende de otros seres humanos, en el establecimiento de interrelaciones, ya que naturalmente convive en grupos $\mathrm{y}$ estructuras sociales, apropiándose de estas experiencias en un contexto histórico que lo determina para incorporar e internalizar así en sus esquemas cognitivos los aprendizajes dados desde las pautas sociales. Claramente uno de los elementos preponderantes de los desarrollos vigotskianos es el carácter innegablemente histórico social del aprendizaje a partir de la apropiación de los elementos culturales que realiza el sujeto. Los colectivos sociales son los que marcan las tendencias educativas del presente y el futuro.
En cuanto al rol del estudiante en educación virtual, Iván Illich citado en Santamaría (2009) nos dice: "podemos dar al aprendiz nuevos enlaces al mundo en lugar de continuar canalizando todos los programas educativos a través del profesor" (p.76). Entonces, sin lugar a dudas la educación virtual debe enfocarse en las relaciones entre los participantes de un proceso formativo, puesto que esto permite mirar los procesos de aprendizaje de una manera diferente a través de las dinámicas globales dadas en las redes sociales que se generan a través de dichos procesos. Tanto las instituciones educativas como los docentes deben ser conscientes de los pasos necesarios a dar y de los cambios que deben incluir hoy en día para responder a las nuevas demandas del contexto y de las personas, valorando más las diferencias individuales, las necesidades de los aprendices y los interesantes procesos y conocimientos que entre ellos se dan, permitiendo así que la educación en verdad avance de lo transmisioncita a lo colaborativo, a lo innovador y lo disruptivo, logrando de esta manera la potenciación del aprendizaje a través del uso de estas redes.

Como no sabemos con certeza cuáles serán las profesiones del futuro, debemos empezar a proyectar desde la incertidumbre, lanzarnos a partir del 
trayecto recorrido y desde lo conocido, pero especialmente de lo desconocido a hacer nuevas propuestas, a escuchar las necesidades del estudiante de hoy, que distan mucho de lo que las instituciones educativas creen. El estudiante se posiciona más desde los colectivos sociales, desde el aprendizaje colaborativo, que de acuerdo con Hernández (2014): "Parece probado que los seres humanos nos agrupamos en comunidades para tratar de alcanzar ciertas metas o ideales. A través de la interacción que requiere el perseguir unos fines comunes, se establecen relaciones que, en gran medida, mantienen unida una colectividad en torno a determinados propósitos, en el caso de las comunidades de aprendizaje, para el logro de los objetivos de aprendizaje". (p. 26). El estudiante actual se percibe $y$ encuentra su identidad como sujeto en red, por lo tanto, las formas de enseñar y aprender deben ya migrar.

En este sentido, autores como Gómez y Gewerc (2002), destacan entre las modificaciones del ámbito educativo, que el hecho de que los roles entre docente y estudiante hayan sido intercambiados provoca un mayor logro del progreso cognitivo mediante la interacción, otorgándole un gran valor pedagógico, con un funcionamiento más de colectivo social a partir de redes y comunidades de aprendizaje autónomas.

Claramente la educación virtual no es mejor que la presencial, ni tampoco está última va a desaparecer por lo menos en el corto tiempo, lo que sucede es que, de acuerdo a los nuevos estilos de vida humana, los colectivos sociales se orientan hacia modalidades a distancia y virtuales que presenciales (Blázquez, 2001). Puesto que las dinámicas de aprendizaje y con construcción de conocimiento se dan más a partir de redes, intereses compartidos, comunidades, que desde la educación formal como se concebía hasta hace poco. Lo que sí es evidente, es que ahora todos los agentes sociales incorporan en mayor o menor medida el uso de TIC para apoyar el desarrollo de sus procesos y facilitar el logro de nuevas rutas de aprendizaje de manera más rápida que lo que le lleva a las instituciones educativas adaptarse (Díaz, Barriga, 2002).

Complementando lo anterior, en el momento en que la educación empezó a incluir TIC, bien fuera como apoyo a la educación tradicional o como un viraje total a procesos de formación virtual sincrónica o asincrónica, cambió rápida y claramente la forma de aprender, puesto que ahora la interacción del estudiante no se da solo con su docente sino también con otras personas de su contexto o de otros 
países, además con diferentes herramientas Web 2.0 que le permiten tener información más actualizada y a la mano de manera constante, y por otro de las redes sociales, que son una nueva forma de comunicación e interrelación mediadas ahora por tecnología. Las redes sociales pueden incluir usuarios de diferentes perfiles $\mathrm{y}$ tener objetivos bastante amplios, como la socialización con conocidos, o conocer nuevas personas, contactos de trabajo, publicación de eventos personales, intercambio de información entre muchos otros, las redes sociales se unen a los procesos formativos, en el punto de intercambio de información, ya que existe actualmente una forma específica de redes sociales, denominadas redes virtuales de aprendizaje, cuyos objetivos son más específicos y se relacionan directamente con compartir información sobre una temática amplia con tópicos específicos, dicha información es proporcionada a la red bien sea por expertos o por cualquier miembro de la red que haya encontrado algo al respecto, las redes sociales de aprendizaje pretenden aparte de compartir información, generar conocimiento, se da un proceso informal de recibir información, reconstruirla, aprender y generar conocimiento. Los colectivos sociales han entendido este potencial de las
TIC en educación y lo están utilizando en beneficio propio.

\section{Conclusión}

Los grupos sociales se están apropiando de la educación virtual y empiezan a generar educación a su medida, autorregulada, modelos disruptivos actuales y cambios, avanzando del fenómeno de los MOOCS, hacia los PLE, la $b$-education, las insignias, y las micro titulaciones. Poco a poco la educación formal y las certificaciones van dejando de ser centrales, abriendo paso al aprendizaje significativo, aprendizaje para toda la vida y cambios sociales desde las dinámicas de los mismos colectivos. Pero mucho más allá, es desde su interior que empiezan a gestarse soluciones reales, creativas, contundentes y antes impensables para el reenfoque de las problemáticas humanas; por todo lo anterior la educación, las instituciones y los agentes educativos del presente debe tomarse muy en serio todo lo nuevo y trascendente que está sucediendo si pretende adentrarse con claridad $y$ pertinencia en el futuro.

La educación y en especial la educación virtual y a distancia hoy más que nunca requieren recorrer nuevos caminos para dar respuestas contundentes y asertivas a las problemáticas humanas actuales, y requiere reconocer que los 
colectivos sociales son los que están reorientando con mucha fuerza los procesos formativos, y por eso debe incluirlos dentro de sus propias dinámicas. Tanto las instituciones como los diferentes agentes sociales $y$ educativos deben avanzar con pasos desde la incertidumbre del hoy hacia la innovación disruptiva que trace los nuevos e impensables enfoques que requiere el mañana, innovación disruptiva entendida como: "Se define a la innovación disruptiva en educación como aquella propuesta que tiene el potencial de impactar a todo el contexto educativo. Su impacto permite que la evolución lineal de un método, técnica o proceso de enseñanza-aprendizaje

cambien

drásticamente alterando la evolución lineal del contexto educativo, modificando permanentemente la forma en la que se relacionan los actores del contexto, los medios y el entorno mismo". (Tecnológico de Monterrey, 2017, s./n.). Lo cual implica nada más y nada menos cambios radicales y verdaderos que avancen desde los temores actuales hacia una educación a distancia y virtuales de punta con innovación estructural, pedagógica, política, académica, pero sobre todo social con la incorporación de los agentes sociales que ya vienen tomando un significativo protagonismo.

\section{Referencias}

Azcárraga, J. (2002). Ciencia y Filosofía. Valencia, España: Universidad de Valencia.

Blázquez, F. (2001). Sociedad de la Información y Educación, Junta De Extremadura, Consejería de Educación, Ciencia y Tecnología, Dirección General de Ordenación, Renovación y Centros. Mérida, España. Recuperado de http://tecnologiaedu.us.es/tecnoedu/images /stories/soc_ed.pdf

Becerra, M. (2002). Apuntes sobre Educación Superior y Virtualidad en la Argentina. Buenos Aires: Universidad Nacional de Quilmes Ediciones.

Burgos, E. (2011). Mediateca escolar: un recurso para la innovación educativa. Editorial Universidad de Burgos. España. Recuperado de: https://ebookcentral.proquest.com/lib/funla

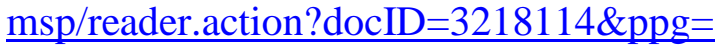
$\underline{1}$

Celaya, J. (2008). La empresa en la web 2.0. Barcelona, España: Ediciones Gestión 2000.

Collis, B. (2001). Linking Organizational Knowledge and Learning. In Montgomerie, C., \& Viteli, J. ED-MEDIA 2001 World Conference on Educational Multimedia, Hypermedia \& Telecommunications. Proceedings (13th, Tampere, Finland, June 25-30, 2001). Association for the Advancement of Computing in Education (AACE), PO Box 3728, Norfolk, VA 23514.

Díaz-Barriga, F. (2002). Estrategias docentes para un aprendizaje significativo: una interpretación constructivista. $2^{\mathrm{a}}$ Edición. México: Mc Graw Hill.

Duart, J., \& Sangrá, A. (2000). Aprender en la virtualidad. Barcelona: Gedisa. 
Frabboni, A., \& Pinto, A. (2006). Introducción a la pedagogía general. México: Siglo XXI editores.

Gómez, S., \& Gewerc, A. (2002): Interacciones entre tutores y alumnos en el contexto de comunidades virtuales de aprendizaje. In II Congreso Europeo de la Información en la Educación y la Ciudadanía: Una Visión Crítica. Barcelona 26, 27 y 28 de Junio.

González, J. (2014). Educación Disruptiva. INED 21. Recuperado de: https://ined21.com/p7137/

Hernández, N. (2014). Planning collaborative learning in virtual environments/ la planificación del aprendizaje colaborativo en entornos virtuales. Comunicar (English Edition), 21(42), 25-32.

Revista Tecnología Social. (2011). ¿Qué es la Tecnología Social? Recuperado de: http://tecnologiasocial.tumblr.com/post/84 77324573/que-es-la-tecnologia-social

Sánchez, U. (2008). Entre la virtualidad y el cara a cara: rastreo de prácticas comunicativas y relacionales generadas por la interacción en Internet para la identificación de nuevos modelos de comunicación. ALAIC. Recuperado de: http://www.alaic.net/alaic30/ponencias/cart as/Internet/ponencias/GT18_9Sanchez.pdf

Santamaría, F. (2009). La web 2.0 como recurso para la enseñanza del francés como lengua extranjera: Redes sociales educativas y comunidades de aprendizaje. Ediciones Gráficas Arial. España.

Silvio, J. (2000). La Virtualización de la Universidad. Cómo transformar la educación superior con la tecnología? Caracas: Instituto Internacional de Educación Superior en América Latina y el Caribe (IESALC)-UNESCO, 10-11.
Suárez,L \& Jardei,O. (2008). Ambientes Virtuales de Aprendizaje Criterios Pedagógicos. Universidad 2008: $6^{\circ}$ Congreso Internacional de Educación Superior. Cuba. Recuperado de: https://ebookcentral.proquest.com/lib/funla msp/reader.action?docID $=3187980 \& p p g=$ 1

Tecnológico de Monterrey. (2017). ¿Qué es innovación educativa?. Observatorio de Innovación Educativa. Recuperado de: https://observatorio.itesm.mx/innovacioned ucativa/

Vygotsky, L. S. (1979). El desarrollo de los procesos psicológicos superiores. Barcelona: Crítica.

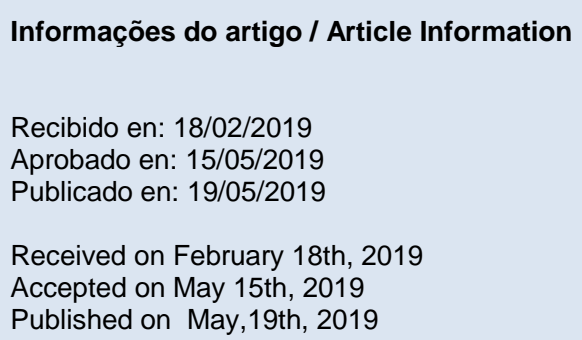

Contribuciones en el artículo: Los autores fueron los responsables de todas las etapas y resultados de la investigación, a saber: elaboración, análisis e interpretación de los datos; escritura y revisión del contenido del manuscrito; aprobación de la versión final que se publicará.

Author Contributions: The authors were responsible for the designing, delineating, analyzing and interpreting the data, production of the manuscript, critical revision of the content and approval of the final version to be published.

Conflictos de interés: Los autores declararon que no había ningún conflicto de interés referente a este artículo.

Conflict of Interest: None reported.

Orcid

Bety Díaz Subieta

(iD) http://orcid.org/0000-0002-9745-0205

Maynor Barrientos Amador

http://orcid.org/0000-0002-5346-3274 


\section{Cómo citar este artículo / How to cite this article}

APA

Subieta, B. D., \& Amador, M. B. (2019). La apropiación de la educación virtual por parte de los colectivos sociales: las nuevas relaciones entre la tecnología, el conocimiento, y lo social. Rev. Bras. Educ. Camp., 4, e6908. DOI: http://dx.doi.org/10.20873/uft.rbec.e6908

ABNT

SUBIETA, B. D.; AMADOR, M. B. La apropiación de la educación virtual por parte de los colectivos sociales: las nuevas relaciones entre la tecnología, el conocimiento, y lo social. Rev. Bras. Educ. Camp., Tocantinópolis, v. 4, e6908,

2019.

DOI:

http://dx.doi.org/10.20873/uft.rbec.e6908 